\title{
Development of Therapeutics for C9ORF72 ALS/FTD-Related Disorders
}

\author{
Maria Sara Cipolat Mis ${ }^{1} \&$ Simona Brajkovic ${ }^{1}$ \& Francesco Tafuri ${ }^{1}$ \& Nereo Bresolin ${ }^{1}$ \& \\ Giacomo P. Comi ${ }^{1} \&$ Stefania Corti ${ }^{1}$
}

\begin{abstract}
The identification of the hexanucleotide repeat expansion (HRE) GGGGCC (G4C2) in the non-coding region of the C9ORF72 gene as the most frequent genetic cause of both amyotrophic lateral sclerosis (ALS) and frontotemporal dementia (FTD) has opened the path for advances in the knowledge and treatment of these disorders, which remain incurable. Recent evidence suggests that HRE RNA can cause gain-of-function neurotoxicity, but haploinsufficiency has also been hypothesized. In this review, we describe the recent developments in therapeutic targeting of the pathological expansion of C9ORF72 for ALS, FTD, and other neurodegenerative disorders. Three approaches are prominent: (1) an antisense oligonucleotides/RNA interference strategy; (2) using small compounds to counteract the toxic effects directly exerted by RNA derived from the repeat transcription (foci), by the translation of dipeptide repeat proteins (DPRs) from the repeated sequence, or by the sequestration of RNA-binding proteins from the C9ORF72 expansion; and (3) gene therapy, not only for silencing the toxic RNA/protein, but also for rescuing haploinsufficiency caused by the reduced transcription of the C9ORF72 coding sequence or by the diminished availability of RNA-binding proteins that are sequestered by RNA foci. Finally, with the perspective of clinical therapy, we
\end{abstract}

Maria Sara Cipolat Mis and Simona Brajkovic contributed equally to this work.

* Stefania Corti stefania.corti@unimi.it

1 Dino Ferrari Centre, Neuroscience Section, Department of Pathophysiology and Transplantation (DEPT), University of Milan, Neurology Unit, IRCCS Foundation Ca' Granda Ospedale Maggiore Policlinico, Via Francesco Sforza 35, 20122 Milan, Italy discuss the most promising progress that has been achieved to date in the field.

Keywords Hexanucleotide repeat expansion . Haploinsufficiency · Antisense oligonucleotides

\section{Introduction}

Amyotrophic lateral sclerosis (ALS) is an incurable and invariably fatal neurodegenerative condition characterized by the progressive loss of both upper and lower motor neurons in the cortex, brainstem, and spinal cord; it clinically results in progressive paralysis and death within 3-5 years of its onset, often due to respiratory failure [1]. No effective therapy is available for this disease. The only drug approved by the FDA and EMA is riluzole, which extends the median lifespan by only 3 months [1]. Thus, the discovery of clinically effective therapies is urgently needed. The vast majority of cases are sporadic (sALS) of unknown origin, while 5-10\% are familial (fALS), often with autosomal dominant inheritance [2]. The disease pathogenesis is multilayered, given that several pathways have been identified as key elements both in the onset and in the progression of the disease. Although several elements have been investigated as possible targets for treatment advancement, the lack of a clear understanding of the causes of ALS, particularly in cases of sALS, has hampered the search for a cure [1]. However, the genetic forms of ALS can offer a solid basis for research since, at least in these cases, the etiopathogenic primum movens is known. The first causative genetic mutations were described in $\mathrm{Cu}-\mathrm{Zn}$ superoxide dismutase 1 (SOD1) gene in 1993 [3], and due to several genome sequencing projects, many of the genes responsible for ALS (>30 genes so far) have been described [4]. The identification of a hexanucleotide repeat expansion (HRE) 
GGGGCC (G4C2 > 30) in the non-coding region of the human chromosome 9 open reading frame 72 (C9ORF72) gene as the most frequent genetic cause of both ALS and frontotemporal dementia (FTD), and which is also present in other neurodegenerative diseases, has represented one of the major breakthroughs in the last 20 years of research in this field $[5,6]$. In fact, the $\mathrm{G}_{4} \mathrm{C}_{2} \mathrm{HRE}$ in the C9ORF72 gene is detected in as many as $40 \%$ of fALS and FTD and in approximately $9 \%$ of sALS cases, with further descriptions in other neurodegenerative disorders [5, 7-9]. This discovery has opened the path for progress in the knowledge and treatment of these devastating diseases. C9ORF72 HRE-linked pathogenesis has been suggested to be elicited by means of loss-offunctionand toxicgain-of-functionmechanisms that comprise the following: (1) transcribed sense GGGGCC exp $_{\text {or antisense }}$ (CCCCGG $\mathrm{Cxp}_{\text {) }}$ RNAs that sequester proteins, therefore interfering with their physiological function [8]; (2) sense or antisense expanded RNAs that are translated via repeatassociated non-AUG initiated (RAN) translation to generate toxic dipeptide repeat proteins (DPRs) [10-13]; or (3) haploinsufficiency that originates from diminished transcription of the C9ORF72 exonic sequence [14, 15] (Fig. 1). It is likely that these mechanisms are not mutually exclusive but correspond to the disease pathogenesis. Indeed, it has been described that HRE RNA forms hairpin and G-quadruplex structures that bind and sequester RNA-binding proteins (RBPs) $[16,17]$. One of the proteins interacting with the $\mathrm{G}_{4} \mathrm{C}_{2}$ RNA is RanGAP1, a key regulator of nucleocytoplasmic transport. RanGAP (an orthologue of human RanGAP1 in Drosophila) physically links with HRE RNA, thereby compromising nuclear import and thus representing a possible central pathogenic event in ALS/FTD [18]. Indeed, DPRs can

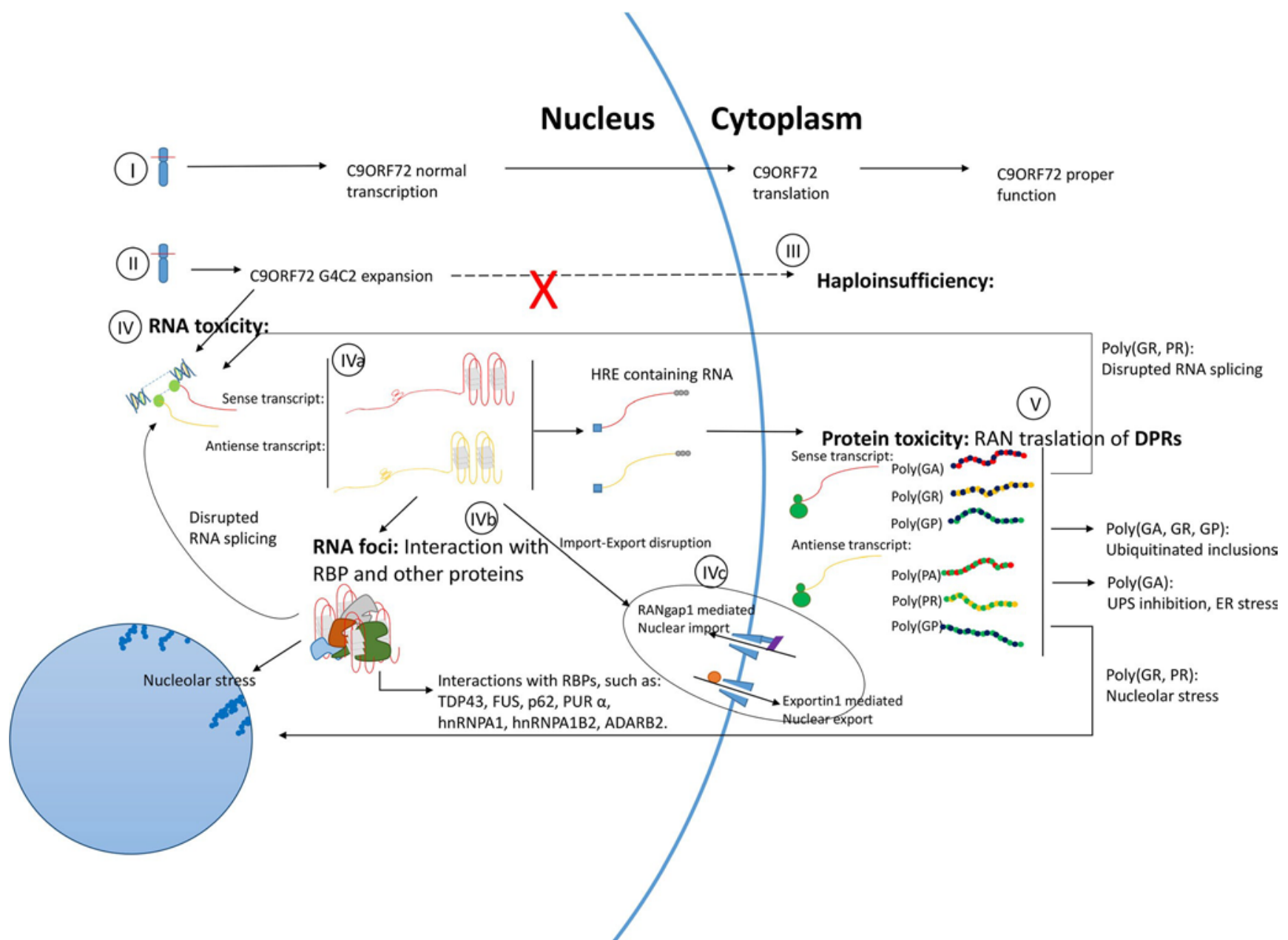

Fig. 1 Schematic representation of C9ORF72 pathogenic events in the nucleus and cytoplasm. I: C9 physiological translation and transcription. II: C9 pathological pathways. III: loss of translation of the C9 hexanucleotide expanded allele, leading to haploinsufficiency and loss of function. IV: RNA toxicity is due to transcription of C9 HRE (hexanucleotide repeat expansion), leading to RNA with a Gquadruplex tertiary structure (IVa), which can form RNA foci $(I V b)$ or interact with nuclear transporters $(I V c)$. RNA foci $(I V b)$ toxic pathways include the disruption of RNA splicing, interactions with RBPs (as TDP43, FUS, p62, PUR $\alpha$, hnRNPA1, hnRNPA1B2, ADARB2), and nucleolarstress. V:Protein toxicity is mediated by cytoplasmic translation of HRE containing RNA, leading to RAN translation of dipeptide repeat proteins (DPRs). The DPR toxic pathways include dipeptide-specific disruption of RNA splicing, nucleolar stress, ubiquitinated inclusions, ubiquitin-proteasome system (UPS) inhibition, and endoplasmic reticu$\operatorname{lum}(\mathrm{ER})$ stress 
also affect the nucleocytoplasmic transport system; this has been demonstrated in yeast and more recently in Drosophila using an RNA interference library that targets proteins of the nuclear pore complex and other regulators, including RanGAP as a toxicity modifier $[19,20]$. Bioinformatic analyses have demonstrated that glycine-arginine and proline-arginine repeats may simulate the nuclear localization signals of these proteins and thus affect nuclear import by overloading the nucleocytoplasmic transport system [20]. These data further establish this secondary event as a possible treatment target.

Overall, the pathways mentioned above may represent possible therapeutic targets in ALS/FTD. If the disease is initiated by RNA foci and/or DPRs originating from the repeated expansion, selectively blocking transcription and/or translation of the repeats might inhibit their negative effects. Remarkable advances have already been achieved in this direction, and they are presented in detail in this review. Briefly, antisense oligonucleotides/RNA interference sequences havebeenused to stimulate the elimination of pathological C9ORF72 transcripts [16, 21, 22] and the small compounds that are able to link the secondary structure of the repeat sequence, which can thereby limit the accumulation of RNA foci and toxic DPRs [23]. Indeed, short hairpin (sh) RNA or similar nucleotidic sequences, either naked or delivered with a viral vector, can be used for the same purpose. However, the haploinsufficiency that is due to reduced transcription of the C9ORF72 coding region or the loss of function of sequestered RBPs can be rescued by gene transfer of wild-type genes. This article highlights the utility of targeting the known C9ORF72 disease pathways at multiple levels (Fig. 2). Furthermore, we discuss the recent therapeutic advances from the perspective of clinical translation.

\section{Oligonucleotide/RNA Interference-Based Therapeutic Strategies}

\section{Antisense Oligonucleotides}

Antisense oligonucleotides(ASOs) are short oligonucleotidic sequences that can interfere with RNA processing/ transduction in many different ways. The consequence of complementary matching can either be the destruction of the messenger RNA (mRNA) of interest with the induction of H/ L RNAse or the prevention of the interaction with RBPs through steric blocking, thereby preventing its splicing/ processing without degradation. Both mechanisms can lead to a decreased level of gene expression for the target protein [16, 24-26]. This strategy is certainly advantageous if the pathogenesis of neurodegeneration is sustained by ва gainof-toxic-function protein^; conversely, if the expansion causes a loss of normal protein activity, this approach may not be completely safe.
There are three C9ORF72 (C9) transcripts in humans: variant 1 (exons 2-5) encoding a 222-aa isoform defined as C9 short (C9-S) and variants 2 and 3 (exons 2-11) encoding a 481-aa isoform defined as C9 long (C9-L) [27]. The HREs are located in the promoter region of variant 2 and the first intron of variants 1 and 3 . The presence of three different C9ORF72 transcripts suggests a need for a selective molecular approach. For example, high levels of the C9ORF72 variant V1 have recently been associated with a longer survival, which suggests that ASOs should not be designed to alter all C9ORF72 expressions [28].

Recent advances in the production of ASO molecules have been made that increase their stability, cell penetration, and targeting of RNA [29]. The feasibility of this approach is supported by data that were obtained from the first clinical trial assessing the safety, tolerability, and pharmacokinetics of ASOs; in this trial, ASOs targeting the SOD1 mRNA (ISIS 333611) were injected intrathecally in mutated ALS subjects [30].

The role of sequestration of RBPs seems to be crucial for the disease pathogenesis, not only in ALS but also in other neuromuscular disorders. Repeat-specific ASOs that are able to block the binding of specific RBPs, preventing their segregation in RNA foci, show therapeutic promise for myotonic dystrophy 1 (DM1), the neuromuscular disease in which the pathological roles of nucleotide repeat expansion and RNA foci were reported for the first time [31]. Treatment with ASOs in in vitro C9ORF72 models showed a positive effect on RNA foci, causing their reduction in fibroblasts and in induced (i) neurons (neurons obtained from direct reprogramming of fibroblasts in patients) derived from C9ORF72-mutated patients [21]. Interestingly, the ASOs tested in these studies [21, 22] were designed to bind DNA sites that were located before and after the repeat, and the molecules formed at both of these sites reduced the presence of RNA foci, even though the binding site after the repeat also led to a depletion of total C9ORF72 RNAs. Regarding the potential effects of completely silencing C9ORF72, studies conducted in adult mice demonstrated that the long-term reduction of C9orf72 RNAs is well tolerated without neuropathological or behavioral defects. However, data obtained in lower species such as zebrafish and in Caenorhabditis elegans suggest that C9ORF72 loss of function can lead to motor defects [14, 32]. These differences could be the consequence of a species-specific tolerance to the deprivation of C9ORF72 homologues or could be the result of dissimilar methods used to decrease the expression of C9ORF72, such as genetic knockdown versus the use of ASOs. A description of a patient carrying a homozygous C9ORF72 repeat expansion who did not show a more severe phenotype suggests that the loss-offunction hypothesis for C9 pathogenesis is less probable or, at least, not the predominant mechanism [33]. The function of the C9ORF72 gene is still largely unknown, so despite the 


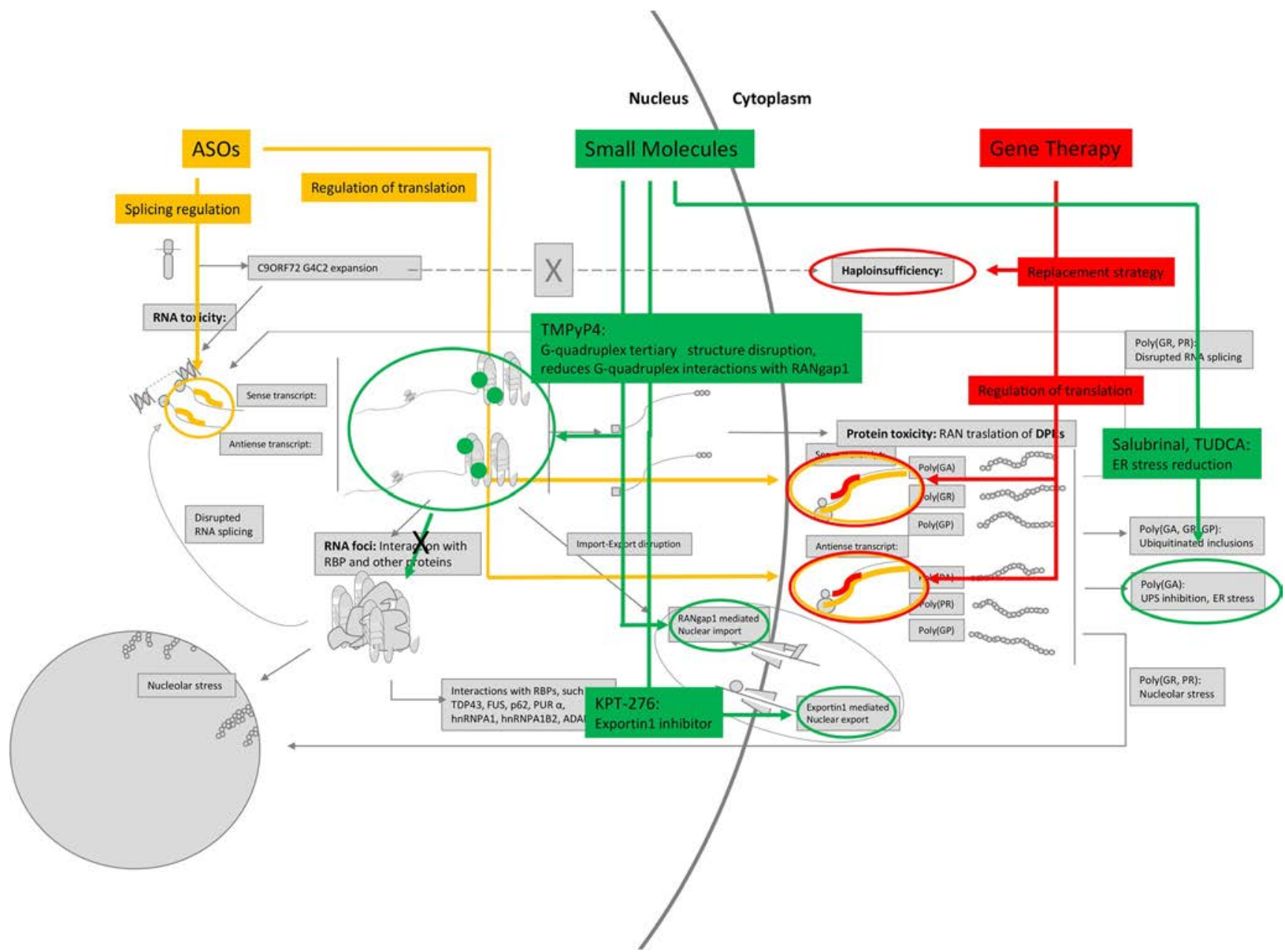

Fig. 2 Schematic representation of the therapeutic approaches and their subcellular interactions. In yellow: antisense oligonucleotide (ASO) therapy is able to act either by regulating splicing or by regulating translation with an RNAse H-dependent pathway or via steric block. In green: small molecules such as TMPyP4 and KPT-276 can be used to restore nuclear cytoplasmic physiological export-import of factors, which appear to be dysregulated in C9 pathology toward an import deficit and an increased export. TMPyP4 can also act by interfering with the formation of an RNA G-quadruplex tertiary structure. Salubrinal and TUDCA can prevent endoplasmic

argument outlined above, the safety of blocking protein expression through ASO-based treatments must be carefully evaluated. The possibility of bidirectional transcription of the repeats should also be considered in the design of ASOs that have to target both sense and antisense strands [21].

In in vivo models of spinal muscular atrophy (SMA), another genetic motor neuron disease, amelioration of the disease phenotype was achieved through the use of ASOs that interact with the splicing regulator molecules, either by blocking splicing suppressors or by promoting splicing activators [34-36]. Clinical trials testing the safety and efficacy of ASOs in humans are in progress to bring this treatment to SMA patients [37]. The results from a phase 1 study of nusinersen (ISIS-SMNRx ASO) in children with SMA have recently been published and support the continued development of this strategy for the treatment of SMA [38].

reticulum (ER) stress, an unfolded protein response, and oxygen radical formation, leading to the inhibition of apoptosis. In red: a gene therapy approach utilizes viral vectors to carry genetic material. It can be used to prevent the unacceptable loss of the C9 mutated allele by replacing the defective one. Gene therapy can also be used to reduce the cytoplasmic translation of HRE containing RNA using an RNA interference (RNAi) strategy. Moreover, it can also be used to perform the so-called Breplacement strategy $\wedge$ to ensure the presence of wild-type transcripts after C9 therapeutic knockout (Color figure online)

Nuclear RNA foci can segregate proteins involved in RNA splicing and regulation, leading to splicing alteration and, consequently, the production of truncated, low-functioning proteins.

So far, splicing alterations have not been clearly recognized in tissues or cells carrying mutated C9ORF72, but the segregation of RBPs in RNA foci provides powerful evidence for an aberrant splicing mechanism in C9ORF72 pathogenesis [16]. Hence, amelioration of the disease phenotype and neuroprotection may be achieved by influencing the pathways involved in the aberrant splicing process.

Recent studies have provided evidence for an HRE RNA gain-of-function mechanism, and to detect which of the candidate RBPs modified G4C2-mediated neurodegeneration, a study in a Drosophila model expressing G4C2 30 repeats 
(G4C2)30 was performed [18]. In this system, the mislocalization of RanGAP leads to an alteration in the nuclear import and this can be preserved and implemented by an interaction between antisense oligonucleotides and small molecules (please see next paragraph) and the HRE G-quadruplexes. These data demonstrate the utility of ASOs in rescuing nucleocytoplasmic transport.

Sense or scrambled ASOs targeting the C9orf72 RNAwere used in C9-ALS reprogrammed neurons to assess whether they were able to restore the observed alteration of the nuclear/cytoplasmic Ran (ras-related nuclear protein) gradient [21, 22]. Ran is a small GTP-binding protein belonging to the RAS superfamily that is crucial for the translocation of RNA and proteins through the nuclear pore complex. Ran is present in the cell as two different nucleotide-bound forms: (1) GDPbound, the form that can enter the nucleus, and (2) GTPbound, the form that can exit the nucleus. The conversion of RanGDP into RanGTP in the nucleus is mediated by the activity of RCC1, the nucleotide exchange factor for Ran. RCC1 is also called Ran guanine nucleotide exchange factor (RanGEF). In the cytoplasm, the intrinsic GTPase activity of Ran that converts RanGTP into RanGDP is activated by binding with Ran GTPase-activating protein (RanGAP) and is facilitated by the link with Ran-binding protein (RanBP). Thus, GTPase activation gives rise to the conversion of RanGTP into RanGDP, closing the вRan nuclear/cytoplasmic cycle^ [21, 22].

In C9ORF72 neurons undergoing sense strand ASO treatment, RNA foci were significantly reduced and the altered nucleocytoplasmic Ran gradient was restored, suggesting that the nucleocytoplasmic transport impairment was caused by C9ORF72 sense strand toxicity [18]. The same cellular models showed an increase of both the nucleocytoplasmic Ran and TAR DNA-binding protein 43 (TDP-43) gradients. In vivo, nuclear import alterations caused by $\mathrm{G} 4 \mathrm{C} 2$ repeats were suppressed by ASOs. In fact, an ASO introduced into the food of Drosophila larvae co-expressing (G4C2)30 and NLS- $\triangle$ NES-GFP (a GFP reporter under nuclear localization signals (NLS) and without nuclear export signals (NES)) decreased the nuclear mislocalization of NLS- $\triangle N E S-G F P$ [18].

Overall, using an ASO strategy, it is possible to positively target the different pathways that are implicated in C9ORF72 disease. Encouraging results have been published with regard to the pharmacological safety and tolerability profile of the ASO approach. An ASO trial against the C9ORF72 expansion is in development and is expected to begin in late 2016. Thus, the next crucial step is to clearly understand the function of the c9orf72 protein and the risks related to its silencing or, as an alternative, to implement ASO strategies that do not cause any modification to the wild-type gene expression to safely translate this approach to the clinic.

\section{RNA Interference Strategy}

An alternative therapeutic approach against RNA-mediated toxicity is represented by the RNA interference (RNAi) strategy [39]. Similar to ASOs, the use of RNAi in the three most common therapeutic approaches (short interference RNAs (siRNAs), shRNAs or artificial microRNAs (miRNAs)) leads to a degradation of RNA transcripts of the target gene. Both the wild-type and the mutant allele are usually targeted without any distinction [39, 40]. Similar to the ASO strategy, this approach appears to be more appropriate in gain-of-function conditions and requires knowledge of the role and the function of the protein because it suggests a potential reduction/loss of protein that could be unsafe for cell survival and normal function. An RNAi approach that is based on targeting a dominant mutant gene has been studied and applied with promising results to other neurodegenerative disorders, such as Parkinson's disease, Alzheimer's disease, and Huntington's disease [40-42]. However, the mutant and normal allele are often quite similar, only differing in a few nucleotides, so the design of an allele-specific RNAi strategy might be difficult. To overcome these difficulties, a Breplacement strategy^ has been proposed (see also the gene therapy paragraph), in which the wild-type gene function is restored by means of designed wild-type genes that are not affected by the activity of RNAi after the inhibition of both mutants and normal alleles using RNAi [43]. Successful results were obtained using this strategy in vitro for other disease genes [43], but further studies are needed to evaluate the effect of the overexpression of the replaced protein as well as the safety and efficacy of the transfection method. In fact, one of the major challenges in the use of the RNAi strategy is how to avoid off-target toxicity that is generally induced by the cellular antiviral response after the delivery of these molecules. Recently, lipid nanoparticles were demonstrated to have high delivery efficiency with little toxicity in the central nervous system (CNS) [44].

\section{Small Molecule Therapeutic Approaches}

Another possible strategy to target the pathogenetic mechanisms of ALS associated with C9ORF72 expansion is based on the use of small molecules and is aimed at reducing the toxicity derived from both RNA foci and RAN translation.

As we mentioned earlier, in repeat expansion diseases, RNA foci are often involved in sequestering cellular proteins, causing their dysfunction. For example, in DM1, the preferential target is muscleblind (MBNL), a splicing modulator. In contrast, C9ORF72-RNA foci seem to bind several proteins that are mostly involved in forming the nuclear pore complex and regulating nucleocytoplasmic trafficking [45]. A recent study suggests that the main C9ORF72 expansion target could 
be RanGAP1, a protein that enhances nuclear import, acting on RanGTPase[18].

In vitro studies have demonstrated that the upregulation of RanGAP and importin $\alpha$ can rescue nucleocytoplasmic trafficking, while the overexpression of proteins such as RanGEF and exportin, which have the opposite activity, can enhance neurodegeneration. In this context, KPT-276 compound, which inhibits exportin 1, improved nuclear import [18]. RanGAP1 has been found to be preferentially bound by sense strand HRE with a G-quadruplex tertiary structure; thus, the cationic 5,10,15,20-tetra( $N$-methyl-4-pyridyl) porphyrin (TMPyP4), a porphyrin that disrupts this type of structure, was successfully applied, reducing RanGAP1 sequestration in RNA foci in vitro and ameliorating nucleocytoplasmic transport in Drosophila model [18]. TMPyP4 has already been shown to destabilize the G-quadruplex of both the DNA and the RNA (CGG)n repeats of the FMR1 gene, which is linked to human pathological conditions such as fragile $\mathrm{X}$ syndrome and fragile $\mathrm{X}$-associated tremor ataxia [46]. TMPyP4 could be particularly important if a specific target of r(GGGGCC)n RNA is not found because it is also able to prevent the sequestration of other proteins, such as hnRNPA1 and ASF/SF2, by C9ORF72-HRE [47].

Another pathological mechanism associated with mutant C9ORF72 is represented by RAN translation leading to DPRs: poly(GA) and poly(GR) from sense G4C2 repeats, poly(PR) and poly(PA) from antisense G2C4 repeats, and poly(GP) proteins from both sense and antisense transcripts [11]. In a Drosophila model expressing RAN, Freibaum and colleagues identified 18 genetic modifiers that belong to the nuclear pore complex. They also observed alteration in the nuclear envelope and RNA nuclear retention [45]. Poly(GA) has been proven to be neurotoxic, being highly aggregation prone and forming ubiquitin and p62-positive cytoplasmic inclusions [48]. Recently, DPR toxicity has been studied in a mouse model, demonstrating that proteins involved in the degradation of the proteasome and in nucleocytoplasmic transport are sequestered by poly(GA). In particular, RanGAP1 was shown to colocalize with poly(GA) inclusions [48]. Inhibiting the ubiquitin-proteasome-system (UPS), RAN poly(GA) indirectly induces endoplasmic reticulum (ER) stress, as marked by PERK-CHOP pathway activation, thus leading to upregulation of the GADD34-PP1C complex and cell death [48]. Salubrinal, an inhibitor of this complex, has been found to reduce ER stress and enhance cell survival in neurons expressing poly(GA). This drug prevents GADD34/protein phosphatase 1 (PP1) from dephosphorylating eukaryotic translational initiation factor $2 \alpha$ (eIF2 $\alpha$ ), which thus reduces protein synthesis and ER stress [49] and inhibits oxidative stress and apoptosis. Salubrinal has been applied with promising results to several medical research fields, in which ER stress is involved, such as brain injury and toxic damage [49-51]. Its neuroprotective activity resulted in the prevention of neuronal cell loss in neurodegenerative processes and has also been tested in in vivo animal models expressing mutant TDP-43 protein, in which the positive effects of salubrinal were not fully explained by its known mechanism of action, thus suggesting other potential pathways for future study [52]. Thus, even if the primary mechanism underlying the cytoprotection by salubrinal seems to be the inhibition of eIF2 $\alpha$ dephosphorylation, other types of pharmacological activity that can lead to off-target effects remain to be identified [53].

Another compound that provides neuroprotection, decreasing ER stress, is tauroursodeoxycholic acid (TUDCA), a chemical chaperone [54]. TUDCA is the taurine conjugate of ursodeoxycholic acid (UDCA); UDCA is approved by the FDA as a treatment for some cholestatic liver diseases and is used in traditional Chinese medicine, where it is obtained from the Asian bear [55]. TUDCA plays an important role in the inhibition of apoptosis due to its activity on mitochondria and unfolded protein responses and its ability to reduce ER stress and oxygen radical formation [56]. For these reasons, TUDCA has been successfully studied as a potential drug for several degenerative pathologies, such as Alzheimer's, Parkinson's, and Huntington's diseases. A recent double-blind placebo-controlled clinical trial led in a cohort of ALS patients has demonstrated a reduction of disease progression in treated subjects [55]. Thus, ALS has been identified as a potential target for TUDCA [56].

The studies about salubrinal and TUDCA in C9ORF72ALS also provide evidence that poly(GA) aggregates can contribute to neurodegeneration, independent of the presence of RNA foci.

Small molecules acting upstream of RAN products were identified as compounds $1 \mathrm{a}, 2$, and 3 and have recently been tested in a study led by Su and colleagues (2014). All three of the molecules were found to reduce RNA foci formation in (G4C2)66 cells. Specifically, compound 3 decreases poly(GP), while both poly(GP) and poly(PR) are markedly reduced by molecules 1a and 2. Moreover, 1a is also active in patientderived i-neurons. Poly(GP) was found to be a detectable marker in C9ORF72-ALS cerebrospinal fluid (CSF), an aspect that could be a useful marker for diagnosis, for correlation studies about disease severity or progression, and for therapy monitoring [23].

Among the DPRs, poly(GR) was the most toxic in both Drosophila and mammalian models [19]. Genetic screening identified several proteins that can rescue DPR toxicity, such as karyopherins, MTR10, which encodes a receptor involved in nuclear import, and NDC1, arelevant component of the nuclear pore complex. In contrast, the authors confirmed that analogues of RanGEF enhance poly(PR) toxicity. Another DPR toxicity modifier is NSR1, the yeast analogue of nucleolin, an important nucleolar protein that binds C9ORF72-HRE, and this in line with the fact that rRNA processing is involved in DPR pathology [17].

Because there is evidence that epigenetic alterations are involved in C9 pathologies, they could be an interesting target 
for small molecules. Infact, all three transcriptional variants of C9ORF72 seem to be reduced in patients' tissues as a possible consequence of the promoter's hypermethylation of $\mathrm{CpG}$ islands and as a result of the HRE's hypermethylation itself in the expanded allele [57-59], thus leading to a potentially deleterious condition of haploinsufficiency. The role of epigenetic processes has already been studied in other repeat expansion disorders, such as Fragile X-associated Tremor/ Ataxia Syndrome (FXTAS), in which the overexpression of FMR1 mRNA can be reversed by histone acetyltransferase (HAT)inhibitors [60]. Zeir and colleagues (2015) have recently studied the activity of bromodomain proteins in C9ORF72ALS. The bromodomain extra terminal (BET) family binds acetylated histones. Specifically, BRD3 was identified as an epigenetic regulator of C9ORF72 expression. Inhibiting BDR3 in C9ORF72-ALS patient fibroblasts resulted in increased levels of the three C9ORF72 transcriptional variants [61]. BRDs share a conserved fold or Bpocket $\wedge$ that comprises a left-handed bundle of four alpha helices ( $\alpha \mathrm{Z}, \alpha \mathrm{A}, \alpha \mathrm{B}, \alpha \mathrm{C})$ that are linked by highly variable loop regions (ZA and BC loops), which form the rim of the substrate-binding pocket and determine the degree of substrate recognition [62]. Among the BET inhibitors, the most studied are JQ1 [63] and IBET-762, both of which interact with the BRD pocket in competition with acetylated peptide binding. However, despite several ongoing clinical trials [64], no BRD inhibitor has so far been approved by theFDA.

Belzil and colleagues (2013) revealed that histone H3 and $\mathrm{H} 4$ in the expanded C9ORF72-allele are trimethylated at lysines 9 (H3K9), 27 (H3K27), 79 (H3K79), and 20 (H4K20) and that the DNA and histone demethylating agent5-aza-2deoxycytidine (5AZA) can increase C9ORF72 mRNA expression[57].

Small molecules represent a key strategy to address the multiple pathogenetic mechanisms of ALS that are associated with the C9ORF72 repeat expansion because they can be specifically targeted, thus leading to a better understanding of the cellularpathological pathways involved, and becausethey can enhance neuroprotection, restoring cell physiological functions. Epigenetics is an interesting field of investigation for discovering potentially characteristic patterns as markers of pathology and for modulating C9ORF72 expression.

\section{Gene Therapy for C9ORF72}

The term gene therapy refers to the cellular delivery of a stable polymer of nucleic acids that modifies the cellular genome by an integrating or non-integrating mechanism, leading to the transcription of new genes. Once translated, the new genes can take part in different pathways according to the therapeutic purpose of the engineered sequence. In this context, for diseases that are characterized by loss of function, it is possible to deliver wild-type genes to supply the missing function of the mutated sequence. In addition, for diseases that are character-

ized by gain of function, it is possible to deliver genes encoding regulatory peptides or nucleotides such as for RNAi. Gene silencing could therefore be combined with a replacement therapy, thus preventing the consequences that may be derived from the total loss of the gene function. This approach is more likely to be suitable in the case of C9ORF72.

One crucial aspect of gene therapy is the delivery of the gene in the CNS using a strategy that is effective but not invasive. Among the DNA-based viral vectors, adenoassociated viruses (AAVs) are a group of single-stranded DNA viruses that are highly promising for use in gene therapy for neurological diseases. In fact, specific serotypes, such as AAV9 and AAV6, can cross the hematoencephalic barrier in vivo after systemic administration, efficiently transfecting neurons and glial cells $[65,66]$. Moreover, AAVs are not associated with human pathology and thus are reasonably safe. The AAV genome is present in the host nuclei in an episomal form, and multiple copies can circularize, thereby enhancing stability and leading to long-lasting and sustained transgene transcription and translation [67, 68].

The main studied AAV serotypes are 2, 6, and 9, and while they share some features, they differ in others [69]. For instance, AAV2 is one of the first and most widely studied, but AAV9 is more widely implemented as a therapy due to its ability to cross the blood-brain barrier after intravenous administration. AAV9 appears to transduce glial cells more than neurons, while AAV6 preferentially transduces neurons [69-71]. Due to their safety and efficacy, many AAV gene therapy clinical trials are ongoing as described at www.clinicaltrials.gov, namely phase I/II for SMA (NCT02122952), Parkinson's disease (NCT01973543, NCT00400634), Alzheimer's disease (NCT00087789), Duchenne muscular dystrophy (NCT00428935), and Leber congenital amaurosis (NCT00749957).

As we mentioned earlier, RNAi molecular therapy represents a possible approach to target some of the pathogenic aspects of disease [39]. Gene therapy can be an effective means to deliver RNAi molecules as well as to achieve permanent gene modulation [71]. AAV can transfer shRNAs, artificial RNAs, or miRNAs aimed at gene silencing to the cells. Among these strategies, miRNAs represent a natural regulatory mechanism through which the cell can regulate its own translation of proteins. In fact, the so-called interference acts in the cytoplasm on the transcribed complementary mRNA ligand, regulating its expression [71, 72].

A recent in vivo study is of particular interest because it provides evidence for a significant delay in disease onset and increased survival in a SOD1-G93A rat model by targeting mutant SOD1 in upper motor neurons by means of an AAV9-shRNA [73]. In general, the use of viral vectors has made the development of gene therapy against mutant SOD1-linked ALS possible, providing encouraging 
results in several studies in which good tolerability profiles were also reported [70, 73, 74].

Gene therapy can also be useful for rescuing C9ORF72 haploinsufficiency or for replacing the RBPs sequestered by toxic RNA foci, such as the following: TDP43, FUS, p62, Pura, hnRNPA1, hnRNPA1B2, and ADARB2 [16, 75, 76].

Additionally, gene therapy can be used to drive the expression of regulators to manage foci formation [77]. This is the case for MBLN1 overexpression in a DM1 disease in vivo model [73] and for Pura in C9ORF72 [78].

In the context of a gene silencing therapy, it is possible that excessive silencing of C9ORF72 will be incompatible with the homeostasis of neuronal cells, so it might be preferable to consider the so-called combined replacement strategy. In this case, non-allele-selective RNAi silencing is used to completely knockout the target gene transcription and translation so that the normal allele can subsequently be replaced [77]. A gene therapy approach using viral vectors can accomplish both of these tasks. Such a therapeutic strategy has been evaluated with a positive outcome in vitro, but preclinical in vivo trials are needed to address potential problems concerning either silencing and gene expression carried out by the replacement strategy or viral delivery and transfection.

The pathogenic elements of C9ORF72 that involve both RNA and protein toxicity interact downstream with a large number of proteins, enzymes, and messengers, many of which have not yet been identified [79].

Although some of these pathogenic elements, such as the sequestration of RBPs, appear to have a critical role in the induction and maintenance of neurotoxicity, it seems unlikely that a gene therapy aimed at restoring only a single transcript will be able to bring an adequate neuroprotection. Rationally, in this context, the targeting of each involved transcript will be necessary to establish neuroprotection.

Given the plethora of pathogenetic events described, a multi-targeted approach that involves different therapeutic strategies should be considered.

Gene therapy may compensate for haploinsufficiency and adjust the translation of the toxic RNA, given that the effectiveness of the miRNAs in the cells requires the presence of preformed machinery, which involves different cytoplasmic enzymes [71, 72, 80]. However, an ASO therapy might be more appropriate to regulate the splicing abnormalities [21, 22] that may contribute to C9ORF72 disease pathogenesis and to provide amelioration of the neuropathological features, neuroprotection, and an improved clinical phenotype.

Another possible gene therapy strategy to achieve C9ORF72 gene correction is the use of the CRISPR/Cas9 type II genome editing system that has recently been developed against several conditions [81]. CRISPR/Cas9 can be designed to cut the repeated expansion of the gene bytargeted homologous recombination. The possibility of achieving a specific targeting of the mutant allele resulting in its direct correction can be achieved, but it is technically challenging. Evaluation of the CRISPR system to determine its therapeutic potential for ALS warrants investigation. Adequate methods of delivery and cell targeting, safety measures, and treatment efficacy are the main goals to pursue and improve in the design and implementation of a gene therapy that might lead to future clinical trials.

\section{Conclusions}

The multiple and complex mechanisms involved in ALS pathogenesis as well as the undefined wild-type and pathological role of C9ORF72 suggest that a successful therapeutic approach should address many targets and pathways. Furthermore, different challenges have to be overcome for the successful implementation of the therapies at the preclinical stages. For instance, novel animal models of the mutant C9orf72 gene have to be validated and used for initial exploration of the therapeutic in vivo effects of the lead compounds, besides the use of human cell lines. Most of the molecular therapies that we described required the implementation of manufacturing technology to produce the amount of product sufficient for large trials and, ultimately, the treatment of many ALS patients. Finally, successful clinical trials would benefit from reliable biomarkers in the design and follow-up. Therefore, C9ORF72 biomarkers should be further investigated.

Until now, the three macro-directions of study that are described in this paper seem to be the most promising and feasible, although clinical trials are yet to start. The positive results obtained in other DNA repeat expansion diseases, such as DM1, and the therapeutic success of some of the biomolecular strategies that we described, such as ASOs and gene therapy in SMA, are stimulating research to move forward in these directions and to share data between researchers investigating different diseases and therapeutic approaches. The ongoing advances made in the field are encouraging, and further focused studies are urgently needed to move them forward even faster to deliver an effective cure for patients.

Acknowledgments Joint Programme Neurodegenerative Disease (JPND) Research grant BDAMNDPATHS^ (2014) and ARISLA grant BSmallRNALS^ (2014) to SC and Italian Ministry of Health RF-2013023555764 and Regione Lombardia TRANS-ALS to GPC are gratefully acknowledged. Wethank Associazione Amici del Centro Dino Ferrarifor their support.

Compliance with Ethical Standards

Conflict of Interest The authors declare that they have no conflicts of interest. 


\section{References}

1. Bucchia M, Ramirez A, Parente V,Simone C, Nizzardo M, Magri F, Dametti S, Corti S(2015)Therapeutic development in amyotrophic lateral sclerosis. Clin Ther37(3):668-80.doi:10.1016/j

2. Renton AE, Chiò A, Traynor BJ (2014) State of play in amyotrophic lateral sclerosis genetics. Nat Neurosci 17(1):17-23. doi:10.1038/nn.3584

3. Rosen DR, Siddique T, Patterson D, Figlewicz DA, Sapp P, Hentati A et al (1993) Mutations in Cu/Zn superoxide dismutase gene are associated with familial amyotrophic lateral sclerosis. Nature 362(6415):59-62

4. Marangi G, Traynor BJ (2015) Genetic causes of amyotrophic lateral sclerosis: new genetic analysis methodologies entailing new opportunities and challenges. Brain Res 1607:75-93. doi:10.1016 /j.brainres.2014.10.009

5. Renton AE, Majounie E, Waite A, Simón-Sánchez J, Rollinson S et al (2011) A hexanucleotide repeat expansion in C9ORF72 is the cause of chromosome 9p21-linked ALS-FTD. Neuron 72(2):25768. doi:10.1016/j.neuron.2011.09.010

6. Souza PV, Pinto WB, Oliveira AS (2015) C9orf72-related disorders: expanding the clinical and genetic spectrum of neurodegenerative diseases. Arq Neuropsiquiatr 73(3):246-56. doi:10.1590 /0004-282X20140229

7. Majounie E, Renton AE, Mok K, Dopper EG, Waite A et al (2012) Frequency of the C9orf72 hexanucleotide repeat expansion in patients with amyotrophic lateral sclerosis and frontotemporal dementia: a cross-sectional study. Lancet Neurol 11(4):323-30. doi:10.1016/S1474-4422(12)70043-1

8. DeJesus-Hernandez M, Mackenzie IR, Boeve BF, Boxer AL, Baker $M$ et al (2011) Expanded GGGGCC hexanucleotide repeat in noncoding region of C9ORF72 causes chromosome 9p-linked FTD and ALS. Neuron 72(2):245-56.doi:10.1016/j.neuron.2011.09.011

9. Ratti A, Corrado L, Castellotti B, Del Bo R, Fogh I et al (2012) C9ORF72 repeat expansion in a large Italian ALS cohort: evidence of a founder effect. Neurobiol Aging 33(10):2528. doi:10.1016/j. neurobiolaging.2012.06.008, e7-14

10. Ash PE, Bieniek KF, Gendron TF, Caulfield T, Lin WL et al (2013) Unconventional translation of C9ORF72 GGGGCC expansion generates insoluble polypeptides specific to c9FTD/ALS. Neuron 77(4):639-46. doi:10.1016/j.neuron.2013.02.004

11. Mori K, Weng SM, Arzberger T, May S, Rentzsch K et al (2013) The C9orf72 GGGGCC repeat is translated into aggregating dipeptide-repeat proteins in FTLD/ALS. Science 339(6125): 1335-8. doi:10.1126/science.1232927

12. Kwon I, Xiang S, Kato M, Wu L, Theodoropoulos P et al(2014) Poly-dipeptides encoded by the C9orf72 repeats bind nucleoli, impede RNA biogenesis, and kill cells. Science 345(6201):1139-45. doi:10.1126/science.1254917

13. Mizielinska S, Grönke S, Niccoli T, Ridler CE, Clayton EL et al (2014) C9orf72 repeat expansions cause neurodegeneration in Drosophila through arginine-rich proteins. Science 345(6201): 1192-4. doi:10.1126/science.1256800

14. Ciura S, Lattante S, Le Ber I, Latouche M, Tostivint H, Brice A, Kabashi E(2013) Loss of function of C9orf72 causes motor deficits in a zebrafish model of amyotrophic lateral sclerosis. Ann Neuro 74(2):180-7. doi:10.1002/ana.23946

15. Gijselinck I, Van Mossevelde S, van der Zee J, Sieben A, Engelborghs S, et al (2015) The C9orf72 repeat size correlates with onset age of disease, DNA methylation and transcriptional downregulation of the promoter. Mol Psychiatry. doi: 10.1038 /mp.2015.159

16. Donnelly CJ, Zhang PW, Pham JT, Haeusler AR, Mistry NA et al (2013) RNA toxicity from the ALS/FTD C9ORF72 expansion is mitigated by antisense intervention. Neuron 80(2):415-28 . doi:10.1016/j.neuron.2013.10.015

17. Haeusler AR, Donnelly CJ, Periz G, Simko EA, Shaw PG et al (2014) C9orf72 nucleotide repeat structures initiate molecular cascades of disease. Nature 507(7491):195-200. doi:10.1038 /nature13124

18. Zhang K, Donnelly CJ, Haeusler AR, Grima JC, Machamer JB et al (2015) The C9orf72 repeat expansion disrupts nucleocytoplasmic transport. Nature525(7567):56-61.doi:10.1038/nature14973

19. Jovičić A, Mertens J, Boeynaems S, Bogaert E, Chai N et al (2015) Modifiers of C9orf72 dipeptide repeat toxicity connect nucleocytoplasmic transport defects to FTD/ALS. Nat Neurosci 18(9):1226-9. doi:10.1038/nn.4085

20. Boeynaems S, Bogaert E, Michiels E, Gijselinck I, Sieben A et al (2016) Drosophila screen connects nuclear transport genes to DPR pathology in c9ALS/FTD. Sci Rep 6:20877. doi:10.1038 /srep20877

21. Lagier-Tourenne C, Baughn M, Rigo F, Sun S, Liu P et al (2013) Targeted degradation of sense and antisense C9orf72 RNA foci as therapy for ALS and frontotemporal degeneration. Proc Natl Acad Sci U S A 110(47):E4530-9. doi:10.1073/pnas.1318835110

22. Sareen D, O’Rourke JG, Meera P, Muhammad AK, Grant S et al (2013) Targeting RNA foci in iPSC-derived motor neurons from ALS patients with a C9ORF72 repeat expansion. Sci TranslMed 5(208):208ra149. doi:10.1126/scitranslmed.3007529

23. Su Z, Zhang Y, Gendron TF, Bauer PO, Chew J et al (2014) Discovery of a biomarker and lead small molecules to target r(GGGGCC)-associated defects in c9FTD/ALS. Neuron 83(5): 1043-50. doi:10.1016/j.neuron.2014.07.041

24. Dias N, Stein CA (2002) Antisense oligonucleotides: basic concepts and mechanisms. Mol Cancer Ther 1(5):347-55

25. Kole R, Krainer AR, Altman S (2012) RNA therapeutics: beyond RNA interference and antisense oligonucleotides. Nat Rev Drug Discov 11(2):125-40. doi:10.1038/nrd3625

26. Riboldi G, Zanetta C, Ranieri M, Nizzardo M, Simone C, Magri F, Bresolin N, Comi GP et al (2014) Antisense oligonucleotide therapy for the treatment of C9ORF72 ALS/FTD diseases. Mol Neurobiol50(3):721-32. doi:10.1007/s12035-014-8724-7

27. Xiao S, MacNair L, McLean J, McGoldrick P, McKeever P, et al., (2016) C9orf72 isoforms in amyotrophic lateral sclerosis and frontotemporal lobar degeneration. Brain Res. Apr 29. doi: 10.1016/j.brainres.2016.04.062

28. van Blitterswijk M, Rademakers R (2015) Neurodegenerative disease: C9orf72 repeats compromise nucleocytoplasmic transport. Nat Rev Neurol 11(12):670-2. doi:10.1038/nrneurol.2015.219

29. Malik R, Roy I (2011) Making sense of therapeutics using antisense technology. Expert Opin Drug Discov 6(5):507-26. doi:10.1517 /17460441.2011.565744

30. Miller TM, Pestronk A, David W, Rothstein J, Simpson E et al (2013) An antisense oligonucleotide against SOD1 delivered intrathecally for patients with SOD1 familial amyotrophic lateral sclerosis: a phase 1, randomised, first-in-man study. Lancet Neurol 12(5):435-42. doi:10.1016/S1474-4422(13)70061-9

31. Mankodi A, Logigian E, Callahan L, McClain C, White R et al (2000) Myotonic dystrophy in transgenic mice expressing anexpandedCUGrepeat. Science 289(5485):1769-73

32. Therrien M, Rouleau GA, Dion PA, Parker JA (2013) Deletion of C9ORF72 results in motor neuron degeneration and stress sensitivity in C. elegans. PLoS ONE 8(12):e83450. doi:10.1371/journal. pone.0083450

33. Fratta P, Poulter M, Lashley T, Rohrer JD, Polke JM et al (2013) Homozygosity for the C9orf72 GGGGCC repeat expansion in frontotemporal dementia. Acta Neuropathol 26(3):401-9. doi:10.1007/s00401-013-1147-0 
34. Porensky PN, Burghes AH (2013) Antisense oligonucleotides for the treatment of spinal muscular atrophy. Hum Gene Ther 24(5): 489-498. doi:10.1089/hum.2012.225

35. Nizzardo M, Simone C, Dametti S, Salani S, Ulzi G, Pagliarani S, Rizzo F, Frattini E et al (2015) Spinal muscular atrophy phenotype is ameliorated in human motor neurons by SMN increase via different novel RNA therapeutic approaches. Sci Rep 5:11746. doi:10.1038/srep11746

36. Nizzardo M, Simone C, Salani S, Ruepp MD, Rizzo F, Ruggieri M, Zanetta C, Brajkovic S et al (2014)Effect of combined systemic and local morpholino treatment on the spinal muscular atrophy delta 7 mouse model phenotype. Clin Ther 36(3):340-56. doi:10.1016/j. clinthera.2014.02.004, e5

37. Faravelli I, Nizzardo M, Comi GP, Corti S (2015) Spinal muscular atrophy—recent therapeuticadvances for an old challenge. Nat Rev Neurol 11(6):351-9. doi:10.1038/nrneurol.2015.77

38. Chiriboga CA, Swoboda KJ, Darras BT, Iannaccone ST, Montes J, De Vivo DC, Norris DA, Bennett CF et al (2016) Results from a phase 1 study of nusinersen (ISIS-SMNRx) in children with spinal muscular atrophy. Neurology 86(10):890-7. doi:10.1212 /WNL.0000000000002445

39. Cooper TA, Wan L, Dreyfuss G (2009) RNA and disease. Cell 136(4):777-93. doi:10.1016/j.cell.2009.02.011

40. Grimm D, Kay MA (2007) Therapeutic application of RNAi: is mRNA targeting finally ready for prime time? J Clin Invest 117(12):3633-3641. doi:10.1172/JCI34129

41. De Ynigo-Mojado L, Martin-Ruiz I, Sutherland JD(2011) Efficient allele-specific targeting of LRRK2 R1441 mutations mediated by RNAi. PLoS ONE 6(6 ):e21352. doi: 10.1371 /journal. pone.0021352

42. Van Bilsen PH, Jaspers L, Lombardi MS, Odekerken JC, Burright EN, Kaemmerer WF (2008) Identification and allele-specific silencing of the mutant huntingtin allele in Huntington's disease patient-derived fibroblasts. Hum Gene Ther 19(7):710-719. doi:10.1089/hum.2007.116

43. Xia XG, Zhou H, Zhou S, Yu Y, Wu R, Xu Z (2005) An RNAi strategy for treatment of amyotrophic lateral sclerosis caused by mutant $\mathrm{Cu}, \mathrm{Zn}$ superoxide dismutase. J Neurochem 92(2):362-367

44. Rungta RL, Choi HB, Lin PJ et al (2013) Lipid nanoparticle delivery of siRNA to silence neuronal gene expression in the brain. Mol Ther Nucleic Acids 2:e136. doi:10.1038/mtna.2013.65

45. Freibaum BD, Lu Y, Lopez-Gonzalez R, Kim NC, Almeida S et al (2015) GGGGCC repeat expansion in C9orf72 compromises nucleocytoplasmic transport. Nature 525(7567):129-33. doi:10.1038/nature14974

46. Weisman-Shomer P, Cohen E, Hershco I, Khateb S, WolfovitzBarchad O et al (2003) The cationic porphyrin TMPyP4destabilizes the tetraplex form of the fragile $\mathrm{X}$ syndrome expanded sequence d(CGG)n. Nucleic Acids Res 31(14):3963-70

47. Zamiri B, Reddy K, Macgregor RB Jr, Pearson CE (2014) TMPyP4 porphyrin distorts RNA G-quadruplex structures of the diseaseassociated r(GGGGCC)n repeat of the C9orf72 gene and blocks interaction of RNA-binding proteins. J Biol Chem 289(8):4653-9. doi:10.1074/jbc.C113.502336

48. Zhang YJ, Jansen-West K, Xu YF, Gendron TF, Bieniek KF et al (2014) Aggregation-prone c9FTD/ALS poly(GA) RAN-translated proteins cause neurotoxicity by inducing ER stress. Acta Neuropathol 128(4):505-24. doi:10.1007/s00401-014-1336-5

49. Boyce M, Bryant KF, Jousse C, Long K, Harding HP et al (2005) A selective inhibitor of eIF2alpha dephosphorylation protects cells from ER stress. Science 307(5711):935-9

50. Rubovitch V,Barak S, Rachmany L, Goldstein RB, Zilberstein Yet al (2015) The neuroprotective effect of salubrinal in a mouse model of traumatic brain injury. Neuromolecular Med 17(1):58-70. doi:10.1007/s12017-015-8340-3
51. Anuncibay-Soto B, Pérez-Rodríguez D, Santos-Galdiano M, Font E, Regueiro-Purriños M, Fernández-López A(2016)Post-ischemic salubrinal treatment results in a neuroprotective role in global cerebral ischemia. J Neurochem. doi:10.1111/jnc.13651

52. Vaccaro A, Patten SA, Aggad D, Julien C, Maios C et al (2013) Pharmacological reduction of ER stress protects against TDP-43 neuronal toxicity in vivo. Neurobiol Dis 55:64-75. doi:10.1016/j. nbd.2013.03.015

53. Matsuoka M, Komoike Y (2015) Experimental evidence shows salubrinal, an eIF2 $\alpha$ dephosphorylation inhibitor, reduces xenotoxicant-induced cellular damage. Int J Mol Sci 16(7): 16275-87. doi:10.3390/ijms160716275

54. Amaral JD, Viana RJ, Ramalho RM, Steer CJ, Rodrigues CM (2009) Bile acids: regulation of apoptosis by ursodeoxycholic acid. J Lipid Res 50(9):1721-34.doi:10.1194/jlr.R900011-JLR200

55. Elia AE, Lalli S, Monsurrò MR, Sagnelli A, Taiello AC et al (2016) Tauroursodeoxycholic acid in the treatment of patients with amyotrophic lateral sclerosis. Eur J Neurol 23(1):45-52. doi:10.1111 /ene.12664

56. Vang S, Longley K, Steer CJ, Low WC (2014) The unexpected uses of urso- and tauroursodeoxycholic acid in the treatment of non-liver diseases. Glob Adv Health Med 3(3):58-69. doi:10.7453 /gahmj.2014.017

57. Belzil VV, Bauer PO, Prudencio M, Gendron TF, Stetler CT et al (2013) Reduced C9orf72 gene expression in c9FTD/ALS is caused by histone trimethylation, an epigenetic event detectable in blood. Acta Neuropathol 126(6):895-905. doi:10.1007/s00401-013-1199-1

58. Xi Z, Zinman L, Moreno D, Schymick J, Liang Y et al (2013) Hypermethylation of the $\mathrm{CpG}$ island near the G4C2 repeat in ALS with a C9orf72 expansion. Am J Hum Genet 92(6):981-9. doi:10.1016/j.ajhg.2013.04.017

59. Xi Z, Zhang M, Bruni AC, Maletta RG, Colao R et al (2015) The C9orf72 repeat expansion itself is methylated in ALS and FTLD patients. Acta Neuropathol 129(5):715-27. doi:10.1007/s00401015-1401-8

60. Todd PK, Oh SY, Krans A, Pandey UB, Di Prospero NA et al (2010) Histone deacetylases suppress CGG repeat-induced neurodegeneration via transcriptional silencing in models of fragile $\mathrm{X}$ tremor ataxia syndrome. PLoS Genet 6(12):e1001240. doi:10.1371/journal.pgen.1001240

61. Zeier Z, Esanov R, Belle KC, Volmar CH, Johnstone AL et al (2015) Bromodomain inhibitors regulate the C9ORF72 locus in ALS. Exp N eurol 271:241 - 50. doi: 10.1016/j. expneurol.2015.06.017

62. Muller S, Filippakopoulos P, Knapp S (2011) Bromodomains as therapeutic targets. Expert Rev Mol Med 13:e29. doi:10.1017 /S1462399411001992

63. Filippakopoulos P, Qi J, Picaud S, Shen Y, Smith WB et al (2010) Selective inhibition of BET bromodomains. Nature 468(7327): 1067-73. doi:10.1038/nature09504

64. Ferri E, Petosa C, McKenna CE (2015) Bromodomains: structure, function and pharmacology of inhibition. Biochem Pharmacol pii S0006-2952(15):00760-1. doi:10.1016/j.bcp.2015.12.005

65. Foust KD, Nurre E, Montgomery CL, Hernandez A, Chan CM, Kaspar BK (2009) Intravascular AAV9 preferentially targetsneonatal neurons and adult astrocytes. Nat Biotechnol 27(1):59-65. doi:10.1038/nbt.1515

66. Zhang H, Yang B, Mu X, Ahmed SS, Su Q, He R, Wang H, Mueller $C$ et al (2011) Several rAAV vectors efficiently cross the bloodbrain barrier and transduce neurons and astrocytes in the neonatal mouse central nervous system. Mol Ther 19(8):1440-1448. doi:10.1038/mt.2011.98

67. Wang D, Gao G (2014) State-of-the-art human gene therapy: part II. Gene therapy strategies and clinical applications. Discov Med 18(98):151-61 
68. Wang D, Zhong L, Nahid MA, Gao G (2014) The potential of adeno-associated viral vectors for gene delivery to muscle tissue. Expert Opin Drug Deliv 11(3):345 -364. doi:10.1517 /17425247.2014.871258

69. San Sebastian W, Samaranch L, Kells AP, Forsayeth J, Bankiewicz KS (2013) Gene therapy for misfolding protein diseases of the central nervous system. Neurotherapeutics 10(3):498-510. doi:10.1007/s13311-013-0191-8

70. Dirren E, Aebischer J, Rochat C, Towne C, Schneider BL, Aebischer P (2015) SOD1 silencing in motoneurons or glia rescues neuromuscular function in ALS mice. Ann Clin Transl Neurol 2(2): 167-84. doi:10.1002/acn3.162

71. Borel F, Kay MA, Mueller C (2014) Recombinant AAV as aplatform for translating the therapeutic potential of RNA interference. Mol Ther 22(4):692-701. doi:10.1038/mt.2013.285

72. Ameres SL, Zamore PD (2013) Diversifying microRNA sequence and function. Nat Rev Mol Cell Biol 14(8):475-88. doi:10.1038 /nrm3611

73. Thomsen GM, Gowing G, Latter J et al (2014) Delayed disease onset and extended survival in the SOD1 $1^{\mathrm{G} 93 \mathrm{~A}}$ rat model of amyotrophic lateral sclerosis after suppression of mutant SOD1 in the motor cortex. J Neurosci 34(47):15587-15600. doi:10.1523 /JNEUROSCI.2037-14.2014

74. Foust KD, Salazar DL, Likhite S, Ferraiuolo L, Ditsworth D, Ilieva H, Meyer K, Schmelzer L et al (2013) Therapeutic AAV9-mediated suppression of mutant SOD1 slows diseaseprogressionand extends survival in models of inherited ALS. Mol Ther 21(12):2148-59. doi:10.1038/mt.2013.211

75. Mori K, Lammich S, Mackenzie IR, Forné I, Zilow S, Kretzschmar H, Edbauer D, Janssens J et al (2013) hnRNP A3 binds to
GGGGCC repeats and is a constituent of p62-positive/TDP43-negative inclusions in the hippocampus of patients with C9orf72 mutations. Acta Neuropathol 125(3):413-23. doi:10.1007/s00401013-1088-7

76. Xu Z, Poidevin M, Li X, Li Y, Shu L, Nelson DL, Li H, Hales CM et al (2013) Expanded GGGGCC repeat RNA associated with amyotrophic lateral sclerosis and frontotemporal dementia causes neurodegeneration. Proc Natl Acad Sci U S A 110(19):7778-83. doi:10.1073/pnas.1219643110

77. Donnelly CJ, Grima JC, Sattler R (2014) Aberrant RNA homeostasis in amyotrophic lateral sclerosis: potential for new therapeutic targets? Neurodegenerative Dis Manag 4(6):417-437. doi:10.2217/nmt.14.36

78. Kanadia RN, Shin J, Yuan Y, Beattie SG, Wheeler TM, Thornton CA, Swanson MS (2006) Reversal of RNA missplicing and myotonia after muscleblind overexpression in a mouse poly(CUG) model for myotonic dystrophy. Proc Natl Acad Sci U S A 103(31):11748-53

79. Mizielinska S, Isaacs AM(2014) C9orf72 amyotrophic lateral sclerosis and frontotemporal dementia: gain or loss of function? Current Opinion in Neurology 27(5):515 - 523. doi: 10.1097 /WCO.0000000000000130

80. Deng Y, Wang CC, Choy KW, Du Q, Chen J, Wang Q, Li L, Chung TK et al (2014) Therapeutic potentials of gene silencing by RNA interference: principles, challenges, and new strategies. Gene 538(2):217-27. doi:10.1016/j.gene.2013.12.019

81. Xiao-Jie L, Hui-Ying X, Zun-Ping K, Jin-Lian C, Li-Juan J (2015) CRISPR-Cas9: a new and promising player in gene therapy. J Med Genet 52(5):289-96. doi:10.1136/jmedgenet-2014-102968 